\title{
Trombolisis y Angioplastía, dos métodos complementarios.
}

\author{
Jorge Bartolucci J.
}

Recibido el 22 de Febrero de 2010, Aceptado el 10 de Marzo de 2010

Rev Chil Cardiol 2010; 29: 94-99

El artículo del Dr. Martínez y cols ${ }^{1 a}$ sobre, "Mortalidad post infarto del miocardio en Chile: Comparación de los registros de angioplastía primaria versus trombolisis", en representación del Registro Nacional de Angioplastía (RENAC) y del Grupo de Estudio Multicéntrico del IAM (GEMI), compara la mortalidad precoz (30 días) y alejada (12-24 meses) de los pacientes post infarto agudo del miocardio (IAM) sometidos a angioplastía primaria (APP) versus los sometidos a trombolisis (TR), evaluando factores que en forma independiente, se asocien con un mayor riesgo de mortalidad, durante los períodos 2003-2004.

En este estudio los autores observan una menor mortalidad de los pacientes sometidos a APP versus los sometidos a TR con estreptokinasa, confirmando la evidencia publicada en la literatura, tanto en estudios preliminares ${ }^{1 b-3}$, así como en los meta-análisis posteriores $^{4-6}$.

En esta muestra se incluyeron 857 pacientes con IAM trombolizados con estreptokinasa y 700 tratados con APP, aunque no hubo diferencias significativas entre los grupos, en relación con las variables estudiadas (edad, sexo, frecuencia de diabetes mellitus, dislipidemia, infarto previo), la mortalidad fue significativamente menor en los pacientes sometidos a APP versus TR, tanto en la mortalidad precoz como la alejada (Intrahospitalaria: $10,6 \%$ vs $6.3 \%$ $p<0,01,30$ días: $11.8 \%$ vs $8.1 \% p<0,01,12$ meses: $15.3 \%$ vs $10.6 \% p<0,001)$. Al igual que en otras comunicaciones previas del registro GEMI, así como en referencias extranjeras, los pacientes más vulnerables resultaron ser los más añosos, el género femenino y la presencia de diabetes mellitus, los cuales tuvieron una menor sobrevida.

Se trata de una muestra muy significativa de pacientes, cuyo análisis cruza a dos importantes registros del Departamento de Estudios Multicéntricos de SOCHICAR, con sólidos datos. Sin embargo, hay algunos elementos en la estructuración del trabajo que vale la pena analizar. Los autores están concientes de estas limitaciones, ya que se trata de un estudio prospectivo y de carácter observacional.

En primer lugar, al no tratarse de un estudio randomizado, ambos grupos en estricto rigor no son comparables, aunque a menudo aceptamos esta metodología comparativa y la utilizamos frecuentemente como un parámetro de referencia. Los resultados similares, aportados por otros estudios durante la generación del conocimiento, fueron randomizados, teniendo la posibilidad de decidir por uno $u$ otro método de reperfusión, dentro del mismo centro, asegurando con esto, que ambos grupos eran comparables. El volver a repetir estudios randomizados de este tipo en nuestro medio, ya sea con estreptokinasa u otros trombolíticos ya estudiados de probada eficacia, en hospitales con capacidad de APP, resulta poco ético, debido a las ventajas de esta última sobre la TR.

Otro hecho que no hace comparable a las dos muestras, es que es altamente probable que el nivel socio económico y de oportunidades de ambos grupos sea substancialmente diferente, lo que conlleva; tiempos de consulta y de atención distintos, desigualdad

Correspondencia: Dr. Jorge Bartolucci Johnston

Unidad de Estudios Cardiológico, Clínica Santa María

Universidad de los Andes. Facultad de Medicina

Fernando Manterola 0530, Providencia

Teléfonos: 9132958 - 9132959

Correo Electrónico: jbartolucci@csm.cl 
en la disponibilidad de fármacos para su tratamiento, atenciones médicas menos frecuentes, probablemente un diferente grado de control de los factores de riesgo cardiovascular, etc., variables que no fueron estudiadas y que dejan un interrogante, sobre un eventual efecto en los resultados.

Lo anterior queda claramente en evidencia en el análisis de los datos, donde la mayoría de los pacientes sometidos a TR fueron provenientes de los hospitales públicos (95.4\%), correspondiendo el $48 \%$ a la Región Metropolitana y los restantes a otras regiones del país. A la inversa, en el caso de los pacientes sometidos a APP, estas fueron realizadas mayoritariamente en los hospitales universitarios, militares y clínicas privadas, sólo un $15 \%$ de las APP se efectuó en un establecimiento público $(40 \%$ en hospitales universitarios, $22 \%$ en hospitales de las fuerzas armadas, $21 \%$ en clínicas privadas, $15 \%$ en hospitales públicos y $2.6 \%$ en otros), realizándose el $88 \%$ de estos procedimientos en la Región Metropolitana.

Otro punto no analizado e imposible de evaluar, por los datos que aportan las fichas de los registro GEMI y RENAC, es la comparación de los tiempos de reperfusión coronaria, tanto para la APP como para TR. Este hecho es de vital importancia para valorar la eficacia del método de reperfusión empleado y no puede ser pasado por alto, ya que podría explicar parte de los resultados. En el trabajo nacional de Grey y colaboradores ${ }^{7}$, efectuado con la base de datos del GEMI, se miden los tiempos de tratamiento para la TR y la APP, en una muestra que incluye solo tres grandes centros, dos públicos y un hospital universitario, siendo mucho menor el tiempo para la TR (41 \pm 45 minutos) en comparación con el de APP (59 \pm 38 minutos), diferencia que fue estadísticamente significativa $(P<0.001)$, con una mortalidad intrahospitalaria para la TR de $10.9 \%$ y de $5.6 \%$ para la APP. En el reciente trabajo GEMI de Prieto y colaboradores ${ }^{8}$, se confirma también que la APP ofrece una menor mortalidad intrahospitalaria en comparación con los pacientes TR.

Diferentes estudios en TR han demostrado que el período que va desde el inicio de los síntomas al reestablecimiento del flujo coronario (tiempo puertaaguja), es la clave que determina la evolución del paciente en el corto y en el largo plazo ${ }^{9,10}$. Cuando comenzamos la terapia fibrinolítica durante la primera hora del inicio de los síntomas, la mortalidad se reduce en un $6.5 \%$, este porcentaje cae entre un 2-3\% después de transcurrido 1-6 horas del inicio de los síntomas ${ }^{11}$. El retardo en la terapia fibrinolítica más allá de los treinta minutos, disminuye gradualmente la eficacia de esta aproximación terapéutica. Efectivamente, de los estudios publicados se desprende que el riesgo de mortalidad al año se incrementa en $7.5 \%$, por cada minuto de retardo en el inicio tratamiento, tanto para la TR como para la APP ${ }^{3,12-14}$. La publicación de Cannon y colaboradores encontró que el tiempo puerta-balón para la APP, especialmente sobre dos horas, estuvo asociado a un mayor incremento de la mortalidad ${ }^{9}$. A pesar de que la evidencia de la reperfusión inmediata muestra una mejoría en la sobrevida, los estudios demuestran que está subutilizada y frecuentemente retardada ${ }^{15}$. Un estudio retrospectivo encontró que menos de la mitad de los pacientes con IAM-SDST tratados entre 19992002, fueron reperfundidos dentro del tiempo puertaaguja recomendado ${ }^{16}$. Adicionalmente datos del National Register of Myocardial Infarction en USA, señalan que menos del $4 \%$ de los pacientes con IAMSDST, tienen un tiempo puerta-balón menor a 90 minutos ${ }^{17}$. Este hecho es tan vital que la AHA Acute Myocardial Infarction Advisory Working Group, trabaja en estrategias que permitan aumentar el número de pacientes IAM-SDST que reciban tratamiento trombolítico.

Un meta-análisis de 23 estudios controlados y randomizados, en los que se compara la APP con la terapia trombolítica, demostró que la APP resultaba en el corto y largo plazo con menos eventos adverso (incluida la muerte ${ }^{4}$. Este aspecto mejora aun más, si los sistemas de transporte a un centro de APP son eficientes ${ }^{18}$. Un análisis agrupado, que incluye 22 estudios, llevados entre 1990 y 2002, demostró una significativa reducción de la mortalidad a 30 días para la AP más que para la terapia trombolítica, indiferente 
del tratamiento ${ }^{26}$. Mientras la ventaja de la APP es clara, la incapacidad para realizarla en sus tiempos y formas, al igual que la reperfusión farmacológica, crea un desafío. La eficacia de ambas terapias de reperfusión disminuye cuando se retarda, esto es especialmente cierto con la terapia fibrinolítica, conduciendo a una clara preferencia a la angioplastía cuando el tiempo de inicio de los síntomas excede las tres horas. La premura en los tiempos se relaciona también con la mortalidad, los primeros 60 minutos son los que acumulan el mayor porcentaje de pacientes fallecidos en el IAM.

Los hechos antes señalados fundamentan la necesidad en la premura de una terapia efectiva. En nuestro medio la consulta es tardía, de acuerdo a los datos del registro GEMI, la consulta se hace alrededor de cuatro horas de iniciados los síntomas, hecho que no ha mejorado en los últimos registros. Agrava esta consideración, que un porcentaje elevado de pacientes no reciben terapia de reperfusión. Antes del GES el 44 $\%$ de los pacientes con IAM-SDST no la recibían, con posterioridad a este la cifra mejora, cayendo al $32 \%{ }^{22}$.

El trabajo de Martínez documentó también tres factores de riesgo independiente de mortalidad, el sexo femenino, la edad avanzada y la presencia de diabetes mellitus.

Respecto a las mujeres, el género femenino mostró una menor sobrevida, este hecho ha sido reiterado en diferentes publicaciones nacionales y extranjeras 8,20,21. El trabajo del GEMI sobre mortalidad intrahospitalaria de acuerdo a género, según la terapia de reperfusión empleada ${ }^{8}$, ofrece claramente un mayor beneficio para los hombres (mortalidad 2.5\%), lo que concuerda con publicaciones internacionales semejantes de gran peso estadístico, por el alto número de APP ${ }^{21}$. Las mujeres en cambio tuvieron una mayor mortalidad (13\%), lo que contrasta con la experiencia del grupo antes mencionado (mortalidad 6\%).

La explicación de esta diferencia está fundamentada por el mayor riesgo basal de las mujeres en el registro GEMI, hecho que se respalda al existir una mayor proporción de Killip III-IV, respecto al registro europeo antes mencionado (13.5\% v/s 6.8\%). Además de lo anterior las mujeres sometidas a APP son de mayor edad, tienen un mayor porcentaje de hipertensión y diabetes, en comparación con los hombres, lo que concuerda con lo publicado por De Luca ${ }^{21}$. Adicional a lo anterior, los hombres reciben una mayor proporción de betabloqueadores, inhibidoras de la ECA y estatinas, fármacos de eficacia demostrada en el IAM. Por lo tanto este mayor perfil de riesgo podría explicar en gran medida la mayor probabilidad de fallecimiento por parte de las mujeres sometidas a APP.

Otro hecho interesante descrito con antelación en los registros GEMI es la alta mortalidad observada en mujeres tratadas con TR, la que supera incluso la mortalidad de las mujeres no sometidas a procedimientos de reperfusión ${ }^{8}$. Estos resultados no tienen por el momento una explicación satisfactoria. El grupo GEMI ha argumentado que las mujeres sometidas a TR son de mayor gravedad que las no tratadas con trombolíticos, que consultan en forma tardía, que hay demora en la decisión terapéutica y que en algunas ocasiones esto pueda deberse a la sintomatología más inespecífica del IAM, que caracteriza a la mujer. Este hecho obliga a estudiar dirigidamente las variables que pueden influir en los resultados adversos, que se observan en este género (la latencia en la consulta, la correcta indicación, el respeto por las contraindicaciones y la calidad de estreptokinasa utilizada, etc.). Prieto señala que este es un nuevo desafió de la red GEMI para optimizar el manejo hospitalario del IAM en nuestros hospitales.

Respecto a la edad avanzada, el estudio de Martínez corrobora el mal pronóstico del paciente añoso como predictor independiente de mortalidad, este hecho también se refuerza en estudios previos desde las primeras publicaciones del GEMI, a mayor edad, mayor riesgo. Los pacientes mayores en Chile tienen una mortalidad tres veces mayor. Este riesgo disminuye, aunque persiste siendo significativo después de ajustarlo por otros factores pronósticos ${ }^{23}$.

De igual manera se documenta en este trabajo un mayor riesgo en presencia de diabetes mellitus (DM), hecho también señalado en trabajos anteriores del 
GEMI $^{24,25}$, donde la mortalidad del paciente con DM era de un $15.3 \%$ y del no diabético de un $8.3 \%$. Tan importante es este factor de riesgo cardiovascular, que en presencia de éste, la mortalidad del hombre se equipara con la de la mujer. Los hombres con DM tienen un riesgo de mortalidad de 1.97 (IC 1.47-2.63) $y$ las mujeres con DM de 1.84 (IC 1.30-2.59). Otro hecho interesante señalado por este grupo es que la mortalidad intrahospitalaria del paciente diabético con IAM-SDST, descendió entre el período 2001 al 2006 del $18.6 \%$ a $10.8 \%{ }^{25}$, atribuido a una mejoría del tratamiento farmacológico y de las estrategias de reperfusión y revascularización.

Desde el 01.07.05, con la implementación de la ley $N^{\circ} 19.966$ que asegura la atención médica a la población, sobre una serie de prestaciones, conocida como "Régimen de Garantías Explícitas en Salud" (Ley GES), se ha dado una amplia cobertura a la atención del IAM en todos los establecimientos del país, independiente de la complejidad de éste, permitiendo la pesquisa y tratamiento trombolítico del IAM-SDST.

La angioplastía primaria (APP) realizada en forma preferencial, se efectúa cada vez más frecuentemente en nuestro medio. Se expandió en su inicio desde el ambiente de los hospitales universitarios a las clínicas privadas, para pasar estos últimos años en forma cada vez más creciente a los hospitales públicos de mayor complejidad. Para ello se han creado turnos de llamada en horarios no funcionario para médicos especialistas y financiado los procedimientos con cobertura estatal.

Se han hecho múltiples esfuerzos en nuestro medio para mejorar el diagnóstico y los tiempos de inicio de la terapia de reperfusión. Han contribuido en esta iniciativa el MINSAL a través de la ley GES, la SOCHICAR con la creación de normas, el GEMI y RENAC a través de su registro nacional y mediante sus publicaciones, la dinámica de mantener reuniones con hospitales de diferente complejidad, que llevan y sostienen el peso de los registros, la publicación de manuales y normas, cursos de capacitación (curso de electrocardiografía y otros), simposio GEMI, el diseño de estrategias para conocer las realidades locales en regiones y hospitales de menor complejidad, el intercambio de información con el MINSAL etc. Todas estas iniciativas han creado conciencia del problema y ayudado a mejorar sus resultados, conduciendo a una reducción de la mortalidad del IAM-SDST, cuya explicación naturalmente es multifactorial. El trabajo de Martínez analiza los registros de los períodos 2003 y 2004, la mortalidad intrahospitalaria por IAM de acuerdo a datos del GEMI, durante esos dos años fue de $10.06 \%$ y $9.52 \%$ respectivamente. Los últimos datos analizados del registro GEMI corresponden al período 2008, la mortalidad en ese año cae $7.78 \%$ (hombres $6.48 \%$, mujeres $11.86 \%$ $\mathrm{p}<0.0001$ ). Datos que indudablemente fundamenta el enorme esfuerzo realizado estos años.

La APP ha sido de gran valor para el paciente en particular y de gran impacto en la sobrevida y el pronóstico de los pacientes con IAM-SDST. Desde la publicación de las guías para el IAM-SDST el 2004, la APP pasó a ser el tratamiento de elección en estos pacientes ${ }^{19}$. Sin embargo, ninguna estrategia de reperfusión aislada resulta conveniente para todos los pacientes, esta es dependiente de cada situación clínica en particular y del momento temporal en que este se encuentre, la elección de la terapia es menos importante que el tiempo perdido en implementar una estrategia de reperfusión. Las guías antes mencionadas proporcionan una serie de factores claves, las cuales debería de ser consideradas cuando se determine una estrategia optima en él paciente individual; hora de inicio de los síntomas, hora de inicio del tratamiento, disponibilidad de laboratorio de caterización cardiaca, habilidad del staff de angioplastía de llamada y posibilidad de eventuales contraindicaciones para el uso de la terapia trombolítica.

Nuestros desafíos futuros están en mejorar los registros, en cuanto a muestras más amplias, que a su vez reflejen realidades locales distintas; la incorporación del registro electrónico en línea, lo 
que permitirá un análisis más expedito de los datos; extender el registro a la mayoría de los pacientes infartados en Chile, en un trabajo conjunto con el MINSAL, idealmente con una ficha única en línea. En relación a la prestación; acortar los tiempos entre el inicio del dolor y la consulta, a través de campañas de educación conjuntas entre el MINSAL, la SOCHICAR y su Fundación Salud Corazón; disponer de trombolíticos de rápido uso y de mayor eficacia
(Tenecteplase); medios de transporte oportunos, rápidos y eficientes a los centros de APP; disponibilidad de equipamiento de APP adecuado y de entrenamiento homogéneo del staff de angioplastía; un seguimiento eficiente de los pacientes post IAM, con exámenes periódicos de la especialidad y terapia eficaz de su enfermedad de base y de los factores de riesgo cardiovascular.

\section{REFERENCIAS:}

1a. MARTÍNEZ A, NAZZAL C, FAJURI A, BARRA L, MAYERSON A, CAVADA G, et. al. Mortalidad post infarto del miocardio en Chile: Comparación de los registros de angioplastía primaria versus trombolisis Rev Chil Cardiol 2010; 29: 29-36

1b. WEABER WD, SIMES RJ, BETRIU A, GRINES CL, ZIJLSTRA F, GARCIA E, et al. Comparison of primary coronary angioplasty and intravenous thrombolytic therapy for acute myocardial infarction a quantitative review. JAMA 1997; 278: 2093-8.

2. The Thrombolysis in Myocardial Infarction (TIMI) trial: Phase I findings. The TIMI Study Group. N Engl J Med 1985; 312: 932-936.

3. An international randomised trial comparing four thrombolytic strategies for acute myocardial infarction. The GUSTO Investigators. N Engl J Med 1993; 329: 673-682.

4. KEELEY EC, BOURA JA, GRINES CL. Primary angioplasty versus intravenous thrombolytic therapy for acute myocardial infarction: a quantitative review of 23 randomised trials. Lancet 2003; 361: 13-20.

5. WIDIMSKÝ P, GROCH L, ZELIZKO M, ASCHERMANN M, BEDNAR F, SURYAPRANATA H. Multicentre randomised trial comparing transport to primary angioplasty vs immediate thrombolysis vs combined strategy for patients with acute myocardial infarction presenting to a community hospital without a catherization laboratory. European Heart Journal (2000) 21, 823-831. do:10.1053/euhj.1999.1993. The European Society of Cardiology.

6. DE LUCA G, DUDEK D, SARDELLA G, MARINO P, CHEVALIER B, ZIJLSTRA F. Adjunctive manual thrombectomy improves myocardial perfusion and mortality in patients undergoing primary percutaneous coronary intervention for ST-elevation myocardial infarction: a meta-analysis of randomized trial. Eur Heart J. 2008; 29: 3002-10. do:10.1093/eurheartj/ehn389. European Society Cardiology.

7. GREIG D, CORBALÁN R, CASTRO P, CAMPOS P, LAMICH R, YOVANINIZ P. Impacto de la trombolisis y de la angioplastía primaria en pacientes con infarto agudo del miocardio tratados en centros hospitalarios terciarios. Rev Med Chile 2008: 136: 1098-1106.

8. PRIETO JC, SANHUEZA C, MARTÍNEZ N, NAZZAL C, CORBALÁNR, CAVADAG, etal. Mortalidad intrahospitalaria en hombres y mujeres según terapias de reperfusión en infarto agudo del miocardio con supradesnivel del ST. Rev Med Chile 2008; 136: 143-150.

9. CANNON CP, GIBSON CM, LAMBREW CT, SHOULTZ DA, LEVY D, FRENCH WJ, et al, Relationship of symptononset-to-ballon time and door-to balloon time with mortality in patients undergoing angioplasty for acute myocardial infarction. JAMA 2000; 283: 2941-2947.

10. BERGER PB, ELLIS SG, HOLMES DR JR, GRANGER CB, CRIGER DA, BETRIU A, et al. Relation between delay in performing direct coronary angioplasty and early clinical outcome in patients with acute myocardial infarction: results from the global use of strategies to open occluded arteries in Acute Coronary Syndromes (GUSTO-Ilb) trial. Circulation 1999; 100: 14-20.

11. O'DONOGHUE M, SABATINE MS. Appropiate invasive and conservative approaches for patients with ST-elevation MI. Curr Treat Opt Cardiovasc Med 2006; 8: 3-11.

12. ZEYMIER $U$, TEBBE $U$, ESSEN R, HAARMANN $W$, NEUHAUS KL. Influence of time to treatment on early infarct-related artery patency after different thrombolytic regimens. ALKK-Study Group. Am Heart J 1999; 137: 3438.

13. DE LUCA G, SURYPRANATA H, ZIJLSTRA F, VAN 'T HOF AW, HOORNTJE JC, GOSSELINK AT, et al. Symptomonset-to-balloon time and mortality in patients with acute myocardial infarction treated by primary angioplasty. J Am Coll Cardiol 2003; 42: 991-997.

14. DE LUCA G, SURYAPRANATA H, OTTERVANGER JP, ANTMAN EM. Time delay to treatment and mortality in primary angioplasty for acute myocardial infarction: every minute of delay counts. Circulation 2004; 109-1223-1225.

15. EAGLE KA, GOODMAN SG, AVEZUM A, BUDAJ A, SULLIVAN CM, LÓPEZ-SENDÓN J; GRACE Investigators. Practice variation and missed opportunities for reperfusion 
in ST-segment-elevation myocardial infarction: findings from the Global Registry of Acute Coronary Events (GRACE). Lancet 2002; 359: 373-377.

16. MCNAMARA RL, HERRIN J, BRADLEY EH, PORTNAY EL, CURTIS JP, WANG Y, et al Hospital improvement in time to reperfusion in patients with acute myocardial infarction, 1999 to 2002. J Am Coll Cardiol 2006; 47: 45-51.

17. NALLAMOTHU BK, BATES ER, HERRIN J, WANG Y, BRADLEY EH, KRUMHOLZ HM; NRMI Investigators. Times to treatment in transfer patients undergoing primary percutaneous coronary intervention in the United States: National Registry of Myocardial Infarction (NRMI)-3/4 analysis. Circulation 2005; 111: 761-767.

18. DALBY M, BOUZAMONDO A, LECHAT P, MONTALESCOT G. Transfer for primary angioplasty versus immediate thrombolysis in acute myocardial infarction: a meta-analysis. Circulation 2003; 108: 1809-1814. Doi: 10.1161/01. Cir.0000091088.63921.8C. American Heart Association.

19. ANTMAM EA, ANBE DT, AMSTRONG PW, BATES ER, GREEN LA, HAND M, et al, ACC/AHA guidelines for the management of patients with ST elevation myocatdial infarction-executive summary. A report of the American College of Cardiology/American Heart Association Task Force on Practice Guidelines. J Am Coll Cardiol 2004; 44: 671-719.

20. TAMIS-HOLLAND JE, PALAZZO A, STEBBINS AL, SLATER JN, BOLAND J, ELLIS SG, et al. Benefits of direct angioplasty for women and men with acute myocardial infarction: results of the global use of strategies to open occluded arteries in acute coronary syndromes (GUSTO II-B) Angioplasty Substudy. Am Heart J 2004; 147: 133-9.

21. DE LUCA G, SURYAPRANATA H, DAMBRINK JH, OTTERVANGER JP, VAN 'T HOF AW, ZIJLSTRA F, et al. Sex-related differences in outcome after ST-segment elevation myocardial infarction treated by primary angioplasty: Data from the Zwolle Myocardial Infarction study. Am Heart J 2004; 148: 852-6.

22. NAZZAL $C$, CAMPOS $P$, CORBALÁN $R$, LANAS $F$, BARTOLUCCI J, SANHUEZA P, et al. Grupo GEMI. Departamento de Estudios Multicéntricos, Sociedad Chilena de Cardiología y Cirugía Cardiovascular. Impacto del plan AUGE en el tratamiento de pacientes con infarto agudo al miocardio con supradesnivel ST, en hospitales chilenos. Rev Méd Chile 2008; 1231-1239.

23. LANAS F, NAZZAL C, CORBALÁN R, PRIETO JC, CHÁVEZ E, BARTOLUCCI J, en representación del grupo GEMI. ¿Es la edad un factor pronóstico independiente en el infarto del miocardio?. Sociedad Chilena de Cardiología y Cirugía Cardiovascular y Grupo GEMI. Rev Chil Cardiol 2004; 23: 262-263.

24. NAZZAL C, CORBALÁN $R$, CAMPOS P, LANAS F, BARTOLUCCI J, PRIETO JC, et al, en representación del grupo GEMI. Diferente riesgo de mortalidad por infarto del miocardio de pacientes con diabetes mellitus según sexo. Rev Chil Cardiol 2004; 23: 290.

25. NAZZAL C, CORBALÁN R, CAMPOS, P, BARTOLUCCI J, CAVADA G, PRIETO JC, et al. In-hospital mortality in diabetic and non-diabetic patients with AMI: a time trend analysis based on the Chilean National Acute Myocardial Infarction Register, 2001-2006. GEMI Group. ESC Congress 2008.

26. BOERSMA E, and The Primary Coronary Angioplasty vs. Tromboysis Group: Does time matter? A pooled analysis of randomized clinical trials comparing primary percutaneous coronary intervention and in-hospital fibrinolysis in acute myocardial infarction patients. Eur Heart J 2006; 27: 779-788. 